\title{
The Clinical Efficacy of Dabrafenib Plus Trametinib Combination Treatment in Stage III/IV BRAF-Mutated Melanoma
}

\author{
Zhao Hongbing ${ }^{1}$, Zhang Xifeng ${ }^{2}$, Feng Ting Ting ${ }^{1}$ and Li Xiao Rui*1 \\ ${ }^{1}$ Department of Oncology, China
}

${ }^{2}$ Shang Qiu Medical School, China

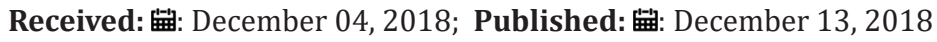

*Corresponding author: Li Xiao Rui, Department of Oncology, Xinxiang, Henan, China

\begin{abstract}
The morbidity of cutaneous melanoma has continued to increase in recent years. Patients with stage III disease are at higher risk for recurrence after locoregional resection and many will ultimately die from metastatic melanoma. BRAF inhibitors vemurafenib and dabrafenib have significantly improved progression-free survival (PFS) and overall survival (OS) as single agents compared with cytotoxic chemotherapy. Acquired resistance to BRAF inhibitors inevitably develops, resulting in a median progression-free survival of 6 to 8 months. Several clinical trials with combination treatment of dabrafenib(150 mg twice daily) plus trametinib(2 mg once daily) in stage III/IV BRAF-mutated melanoma have been reported. These clinical trials identify combination treatment of dabrafenib plus trametinib as front-line therapy in stage III/IV BRAF-mutated melanoma.
\end{abstract}

Keywords: Dabrafenib; Trametinib; Braf; Mek; Mutant; Melanoma

\section{Mini Review}

The morbidity of cutaneous melanoma has continued to increase in recent years [1]. Patients with stage III disease are at higher risk for recurrence after locoregional resection and many will ultimately die from metastatic melanoma [2]. Oncogenic driver mutations in BRAF are found in approximately $40 \%$ to $50 \%$ of cutaneous melanomas and induce constitutive activation of the MAPK signaling pathway, driving melanoma growth and progression [3]. BRAF inhibitors vemurafenib and dabrafenib have significantly improved progression-free survival (PFS) and overall survival (OS) as single agents compared with cytotoxic chemotherapy [4,5]. Despite these advances, acquired resistance to BRAF inhibitors inevitably develops, resulting in a median progression-free survival of 6 to 8 months [6].
Mechanisms of acquired resistance include secondary NRAS or MEK mutations [7]. In preclinical models, combined BRAF and MEK inhibition achieves more via abrogation of MAPK signaling, thereby forestalling the development of acquired resistance and suppressing paradoxic activation of the MAPK pathway [8-14]. Several clinical trials with combination treatment of dabrafenib(150mg twice daily) plus trametinib(2mg once daily) in stage III/IV BRAFmutated melanoma have been reported. Here, we review the clinical efficacy of these clinical trials. (Inclusion criteria for these studies included age $\geq 16$ years, histologically confirmed BRAFV600E- or BRAFV600K-mutant melanoma, Eastern Cooperative Oncology Group (ECOG) performance status (PS) of 0, 1, or 2.)

Table 1: The Fundamental Parameters and Efficacy of Clinical Trials.

\begin{tabular}{|c|c|c|c|c|c|c|c|}
\hline Clinical Trial Information & Phase & Stage & Patients Status & $\begin{array}{l}\text { ECOG } \\
\text { PS }\end{array}$ & $\begin{array}{l}\text { Prior } \\
\text { BRAFi }\end{array}$ & ORR(\%) & Efficacy \\
\hline NCT01072175 [9] & phase I/II trial & IIIc,IV & $\mathrm{PD}$ & 0 to 1 & Yes & $\begin{array}{l}\text { CohortB:15; } \\
\text { CohortC:13 }\end{array}$ & yes \\
\hline NCT01619774 [10] & Phase 2 trial & IIIc,IV & refractory & 0 to 2 & yes & 10 & yes \\
\hline NCT02039947 [11] & phase 2 trial & III,IV & brain metastases & 0,1 , or 2 & no & $\begin{array}{c}\text { intracranial } \\
\text { response:44-59 }\end{array}$ & yes \\
\hline
\end{tabular}




\begin{tabular}{|c|c|c|c|c|c|c|c|}
\hline NCT02296996 [12] & phase 2 trial & IIIc,IV & PD & 0, 1, or 2 & yes & 32 & $\begin{array}{c}\text { Yes(Re- } \\
\text { challenge) }\end{array}$ \\
\hline NCT01597908 [13] & phase 3 trial & IIIc,IV & untreated patients & 0 or 1 & no & 64 & yes \\
\hline NCT01682083 [14] & phase 3 trial & III & Complete resected & 0 or 1 & no & 3-year OS rate:86 & yes \\
\hline
\end{tabular}

\section{Result}

Table 1 the fundamental parameters and efficacy of clinical trials.

\section{Conclusion}

These clinical trials identify combination treatment of dabrafenib(150mg twice daily) plus trametinib(2 mg once daily) as front-line therapy in stage III//IV BRAF-mutated melanoma.

\section{References}

1. (2017) National Comprehensive Cancer Network. Clinical practice guidelines in oncology melanoma. V1.

2. Siege RL, Miller KD, Jemal A (2017) Cancer statistics. CA Cancer J Clin 67(1): 7-30.

3. Long GV, Menzies AM, Nagrial AM, Haydu LE, Hamilton AL, et al. (2011) Prognostic and clinicopathologic associations of oncogenic BRAF in metastatic melanoma. J Clin Oncol 29(10): 1239-1246.

4. Chapman PB, Hauschild A, Robert C, John B Haanen, Paolo Ascierto, et al. (2011) Improved survival with vemurafenib in melanoma with BRAF V600E mutation. N Engl J Med 364(26): 2507-2516.

5. Hauschild A, Grob JJ, Demidov LV, Jouary T, Gutzmer R, et al. (2012) Dabrafenib in BRAF-mutated metastatic melanoma: a multicentre, openlabel, phase 3 randomised controlled trial. Lancet 380(9839): 358-365.

6. Solit DB, Rosen N (2011) Resistance to BRAF inhibition in melanomas. N Engl J Med 364(8): 772-774.

7. Nazarian R, Shi H, Wang Q Kong X, Koya RC, et al. (2010) Melanomas acquire resistance to B-RAF(V600E) inhibition by RTK or N-RAS upregulation. Nature 468(7326): 973-977.

\section{ISSN: 2574-1241}

DOI: $10.26717 / B J S T R .2018 .12 .002205$

Li Xiao Rui. Biomed J Sci \& Tech Res

CC (i) This work is licensed under Creative

BY Commons Attribution 4.0 License

Submission Link: https://biomedres.us/submit-manuscript.php
8. Shi H, Moriceau G, Kong X, Lee MK, Lee H, et al. (2012) Melanoma wholeexome sequencing identifies (V600E)B-RAF amplification-mediated acquired B-RAF inhibitor resistance. Nat Commun 3: 724.

9. Johnson DB, Flaherty KT, Weber JS, Jeffrey R Infante, Kevin B Kim, et al. (2014) Combined BRAF (Dabrafenib) and MEK Inhibition (Trametinib) in Patients with $\mathrm{BRAF}^{\mathrm{V} 600}$-Mutant Melanoma Experiencing Progression with Single-Agent BRAF Inhibitor. J Clin Oncol 32(33): 3697-3704.

10. Chen G, Mc Quade JL, Panka DJ, Hudgens CW, Amin Mansour A, et al. (2016) Clinical, Molecular, and Immune Analysis of DabrafenibTrametinib Combination Treatment for BRAF Inhibitor-Refractory Metastatic Melanoma A Phase 2 Clinical Trial [J]. JAMA Oncol 2(8): 10561064.

11. Davies MA, Saiag P, Robert C, Grob JJ, Flaherty KT, et al. (2017) Dabrafenib plus trametinib in patients with $\mathrm{BRAF}^{\mathrm{V} 600}$-mutant melanoma brain metastases (COMBI-MB): a multicentre, multicohort, open-label, phase 2 trial. Lancet Oncol 18(7): 863-873.

12. Schreuer M, Jansen Y, Planken S, Chevolet I, Seremet T, et al. (2017) Combination of dabrafenib plus trametinib for BRAF and MEK inhibitor pretreated patients with advanced $\mathrm{BRAF}^{\mathrm{V} 600}$-mutant melanoma: an open-label, single arm, dual-centre, phase 2 clinical trial. Lancet Oncol 8(4): 464-472.

13. Robert C, Karaszewska B, Schachter J, Piotr Rutkowski, Andrzej Mackiewicz, et al. (2015) Improved Overall Survival in Melanoma with Combined Dabrafenib and Trametinib. N Engl J Med 372(1): 30-39.

14. Long GV, Hauschild A, Santinami M, Victoria Atkinson, Mario Mandalà, et al. (2017) Adjuvant Dabrafenib plus Trametinib in Stage III BRAFMutated Melanoma. N Engl J Med 377(19): 1813-1823.

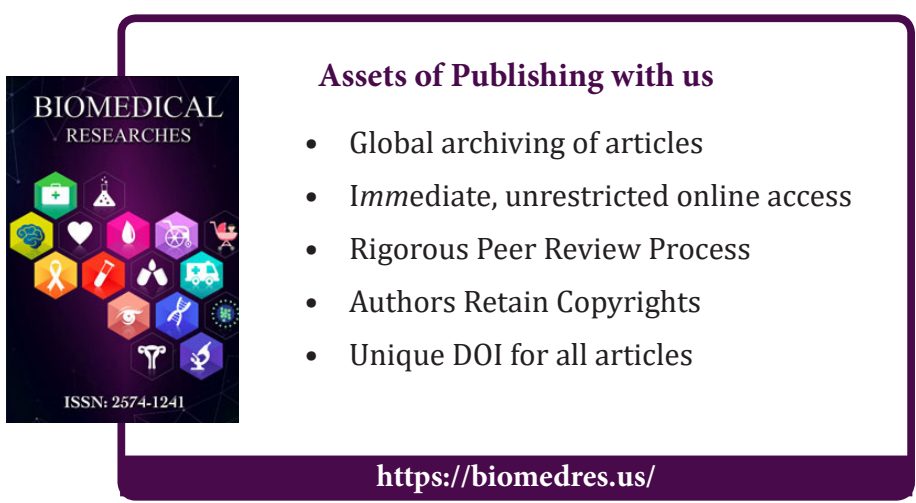

